\title{
Narrow-band imaging and white-light endoscopy with optical magnification in the diagnosis of dysplasia in Barrett's esophagus: results of the Asia-Pacific Barrett's Consortium
}

Authors

Institutions

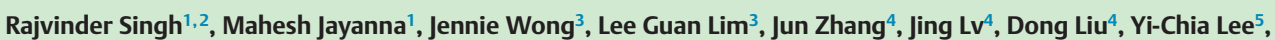
Ming-Lun Han ${ }^{5}$, Ping-Huei Tseng ${ }^{5}$, Vikneswaran Namasivayam ${ }^{6}$, Rupa Banerjee ${ }^{7}$, Noriya Uedo ${ }^{8}$, Wah Kheong Chan ${ }^{9}$, Shiaw Hooi Ho ${ }^{9}$, Shi-yao Chen ${ }^{10}$, Shobna Bhatia ${ }^{11}$, Kohei Funasaka ${ }^{12}$, Takafumi Ando ${ }^{13}$, Justin $\mathbf{W u}^{14}$, Cosmas Lesmana ${ }^{15}$, William Tam ${ }^{1,2}$, Wen-Lun Wang ${ }^{16}$, Chi-Yang Chang ${ }^{16}$, Hwoon-Yong Jung ${ }^{17}$, Kee Wook Jung ${ }^{17}$, Muhammad Begawan Bestari $^{18}$, Kenshi Yao ${ }^{19}$, Vui Heng Chong ${ }^{20}$, Prateek Sharma ${ }^{21}$, Khek-Yu Ho ${ }^{3}$ submitted

23. February 2014 accepted after revision 24. June 2014

\section{Bibliography}

DOI http://dx.doi.org/

10.1055/s-0034-1377610

Published online: 26.9.2014

Endoscopy International Open

2015; 03: E14-E18

(c) Georg Thieme Verlag KG

Stuttgart · New York

E-ISSN 2196-9736

Corresponding author

Rajvinder Singh, MBBS MRCP MPhil FRACP AM FRCP

Clinical Associate Professor

Head of Endoscopy

Lyell McEwin Hospital \&

University of Adelaide

Adelaide

Australia

Fax: +61-8-81829837

rajvinder.singh@health.sa.gov. au
Objective: The advent and utility of new endoscopic imaging modalities for predicting the histology of Barrett's esophagus (BE) in real time with high accuracy appear promising and could potentially obviate the need to perform random biopsies where guidelines are poorly adhered to. We embarked on evaluating the performance characteristics of white-light endoscopy with magnification (WLE-Z), narrow-band imaging with magnification (NBI-z) and a combination of both modalities.

Design: This was a prospective online study with 28 endoscopists from 11 countries (Asia-Pacific region) participating as assessors. In total, 35 patients with $\mathrm{BE}$ were assessed using 150 slides from WLE-z and NBI-z randomly arranged using a simple classification with corresponding histology. The overall Accuracy (Acc), Sensitivity (Sn), Specificity (Sp), Positive Predictive Value (PPV),

\section{Introduction}

Barrett's esophagus (BE) is the only known premalignant precursor of esophageal adenocarcinoma, a tumor that is rapidly increasing in the developed world [1]. At present, endoscopic surveillance with white-light endoscopy (WLE) and random 4-quadrant biopsies is considered the standard of care for detecting intestinal metaplasia and neoplasia in BE. However, current recommended guidelines for surveying patients with $\mathrm{BE}$ are time consuming and poorly adhered to [2]. Over the last decade, new endoscopic imaging technologies have shown great promise in detecting dysplasia and early neoplasia in BE [3-8]. Histology can be predicted with high accuracy potentially making surveillance cost-effective. Recent data have emerged that a targeted biopsy approach using Narrow-Band Imaging (NBI) could be considered in patients undergoing surveillance for BE [9]. Given the low prevalence in $\mathrm{BE}$, endoscopists from the Asia Pacific region may be less experienced with and Negative Predictive Value (NPV) of WLE-Z, NBI-Z and a combination of both were calculated. Results: The overall Acc for WLE-z and NBI-z images was $87.1 \%$ and $88.7 \%$, respectively. When images from the two modalities were placed side by side, the Acc increased to $90.3 \%$. The Sn, Sp, PPV, and NPV of WLE-z were $48 \%, 92 \%, 45 \%$, and $93 \%$ while with NBI-z, these improved to $89 \%, 89$ $\%, 56 \%$, and $98 \%$, respectively. When both imaging modalities were viewed together, they improved further to $93 \%, 90 \%, 61 \%$, and $99 \%$.

Conclusion: The high NPV (99\%) when both WLE$\mathrm{Z}$ and NBI-Z were used simultaneously indicates that areas with regular appearance that are diagnosed with confidence can effectively be left alone and not biopsied when performed at a skilled resourced center. This approach could potentially lead to a paradigm shift of how patients with $\mathrm{BE}$ are assessed.

this condition. On the other hand, they may be well versed with image enhancing endoscopic modalities given the high prevalence of gastric cancers, especially in East Asia. We embarked on a study to evaluate the performance characteristics of WLE with optical magnification (WLE-z), NBI with optical magnification (NBI-Z) and a combination of both modalities in the assessment of patients presenting for surveillance endoscopy for $\mathrm{BE}$ amongst a large group of endoscopists from the Asia Pacific region.

\section{Methods}

$\nabla$

A total of 28 endoscopists from 11 countries (Asia-Pacific Barrett's Consortium) who have an interest in BE were invited to participate in an online study. Fifty images of WLE- $z$ and 50 corresponding images of $\mathrm{NBI}-\mathrm{Z}$ in $\mathrm{BE}$ with corresponding histopathology were prospectively collected. 


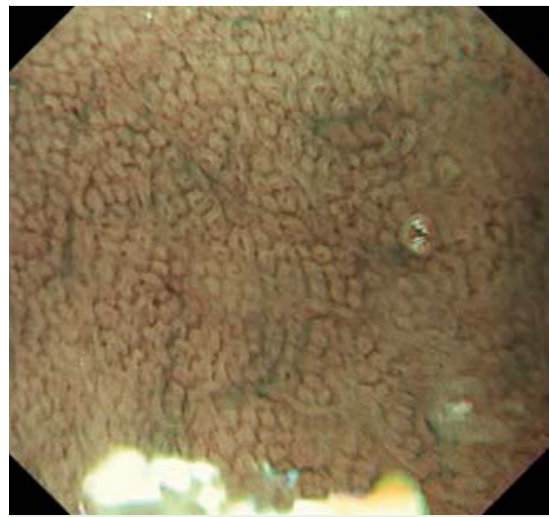

Fig. 1 Round pits and regular microvasculature (no dysplasia).

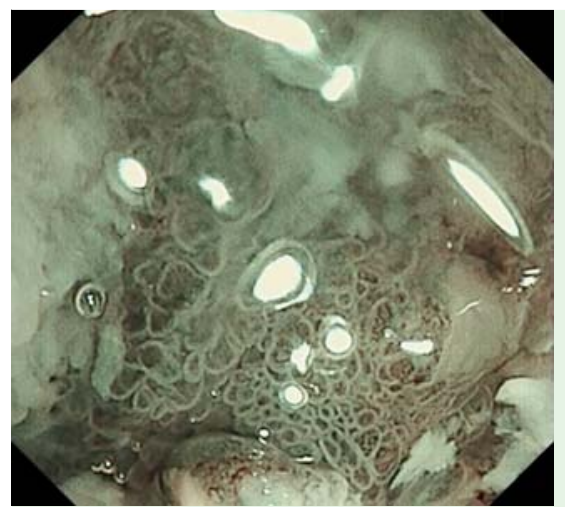

Fig.4 Irregular/absent pits and irregular microvasculature (high grade dysplasia).

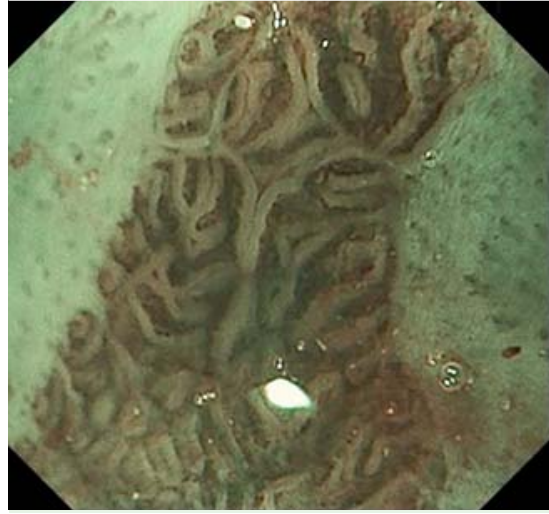

Fig. 2 Ridge/villous pits and regular microvasculature (no dysplasia)

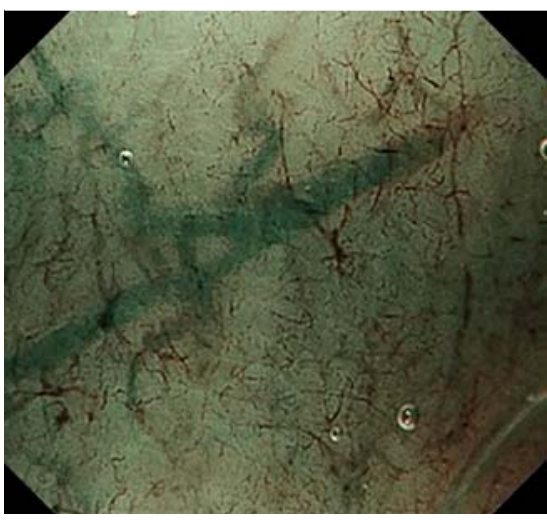

Fig. 3 Absent pits and regular microvasculature (no dysplasia).

\section{Image acquisition}

All images were taken by a single gastroenterologist (RS) with expertise in advanced endoscopic imaging at a single Australian center, which has the required equipment. All procedures were performed using the commercially available Olympus $160 \mathrm{Z}$ endoscope with optical magnification $(\times 115)$ capability. A transparent cap was used and adjusted to a length of $2 \mathrm{~mm}$ at the tip of the endoscope. After obtaining informed consent, all patients were offered local pharyngeal anesthesia with xylocaine spray followed by induction and maintenance of deep sedation with intravenous Midazolam and Propofol. It is imperative to visualize the mucosa clearly, hence patients were given a mucolytic agent, $\mathrm{N}$-acetylcysteine, and a defoaming agent, Simethicone, mixed with water $(50 \mathrm{~mL})$ to drink before the procedure. Following intubation of the esophagus, an additional $10-20 \mathrm{~mL}$ of the mucolytic agent was flushed to rid the surface of any adherent mucus. This was followed by flushing the surface with dilute adrenaline $(1: 20000)(10-20 \mathrm{~mL})$ to reduce interference with blood and im-

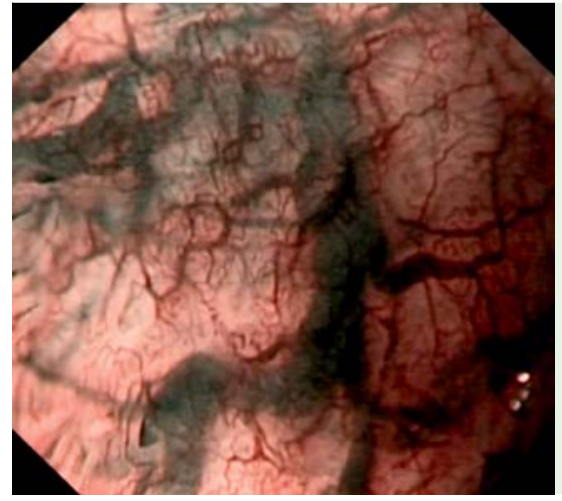

Fig. 5 Dilated vasculature but no change in caliber (equivocal). prove mucosal visualization after biopsies had been taken. To minimize movement artifacts and, if deemed necessary by the endoscopist, sedation was titrated further. If the esophagus exhibited excessive peristaltic activity, an antispasmodic agent, hyoscine- $N$-butylbromide (Buscopan, $10-20 \mathrm{mg}$ ), was given intravenously.

The esophagus was first examined in the overview mode (without activation of NBI or optical magnification function). The endoscopist recorded the length of Barrett's segment according to Prague's C\&M criteria and any macroscopically evident lesions. Only inconspicuous, flat areas or areas adjacent to macroscopically visible lesions in the Barrett's segment were further assessed in the NBI- $z$ mode. We ensured that the imaged view was the area biopsied by applying light suction pressure to the mucosa, thus enabling the cap to fix to the imaged area. All images were then stored as high definition JPEG files (200-300 kb, $1280 \times 1024$ pixel array, 32-bit color). Corresponding biopsies of each imaged area were placed in separate labeled pots filled with $10 \%$ buffered formalin and sent for histopathological analysis by a single gastrointestinal pathologist with expertise in Barrett's esophagus. Only clear images with corresponding histopathology were selected. A total of 100 images were obtained from 35 patients.

\section{Learning phase}

A short learning Microsoft PowerPoint presentation was given to all of the endoscopists at the Asia Pacific Digestive Week 2011 held in Singapore. This session lasted for 1 hour. In addition, a total of 30 sample images were shown to the participants. They were also given sample PowerPoint slides as a guide after the education session. Subsequently, all participants were invited to predict the histology of the images, which were presented on a web portal for direct online assessment. 


\section{Classification}

A simplified classification based on the microstructural pattern and microvascular architecture was used to grade the images:

(1) Regular pits and/or vasculature (R): no dysplasia ( $\mathbf{~ F i g . ~} \mathbf{1 - 3}$ ) (absent/round/oval/linear/cerebriform/villous pits with regular vasculature).

(2) Irregular/absent pits and irregular vasculature (IR): high grade dysplasia ( $\bullet$ Fig. 4 ).

(3) Equivocal (E): not clear/unsure. Area may exhibit dilated vasculature but no change in caliber $(\boldsymbol{Q}$ Fig. 5 ).

(1) and (2) were graded with confidence whereas (3) was graded no confidence.

\section{Image evaluation}

The 100 images were then randomly mixed and displayed as 100 single images on an online portal which consisted of three parts: i) Part-I: participant's information, ii) Part-II comprising of 100 WLE-z/NBI-z images, and iii) Part-III comprising of 50 NBI-z images placed alongside the corresponding WLE-z images. This last exercise was done to simulate real-time endoscopy where both imaging modalities are often interchanged. No clinical data was revealed to the evaluators. The endoscopists were given specific instructions to complete the study within 1 month of the learning session (online portal webpage was closed thereafter). The overall Accuracy (Acc), Sensitivity (Sn), Specificity (Sp), Positive Predictive Value (PPV), and Negative Predictive Value (NPV) of WLE- $z$, NBI-z and the combination were then determined.

\section{Results}

$\nabla$

\section{Endoscopist characteristics}

A total of 28 endoscopists representing 11 countries in the Asia Pacific region with a diverse range of experience participated in the study. Twelve ( $43 \%$ ) had more than 10 years of experience as a consultant, $3(11 \%) 8-10$ years, 2 (7\%) $5-7$ years, $6(21 \%) 2-4$ years and $5(18 \%)$ less than 2 years. The number of endoscopies performed by the evaluators ranged from 500 to 3000 per year with $4(14 \%)$ doing more than 3000 procedures per year and 5 (18\%) performing less than 500 per year. $\bigcirc$ Table 1 summarizes the average number of $\mathrm{BE}$ seen by endoscopists per year.

\section{Image evaluation: overall}

A total of 4200 images were available for evaluation (28 endoscopists; 150 images each). These images were obtained from 43 non-dysplastic and 7 dysplastic areas. The assessors were able to grade 3370 images with confidence (80.2\%). The overall Acc for WLE-z and NBI-z images were $87.1 \%$ and $88.7 \%$, respectively. When images from the two modalities were placed side by side, the Acc increased to $90.3 \%$. The Sn, Sp, PPV, and NPV of WLE-z were $48 \%, 92 \%, 45 \%$, and $93 \%$, while with NBI-z, these values improved to $89 \%, 89 \%, 56 \%$, and $98 \%$, respectively. When both imaging modalities were viewed together, these values improved further to $93 \%, 90 \%, 61 \%$, and $99 \%$ ( $\bullet$ Table 2 ).

\begin{tabular}{|l|l|}
\hline Average number of BE seen/year & Number \\
\hline$<20$ & 19 \\
\hline $21-50$ & 5 \\
\hline $51-100$ & 3 \\
\hline$>100$ & 1 \\
\hline
\end{tabular}

Table 1 Average number of Barrett's esophagus (BE) patients seen yearly by assessors.

Table 2 Sensitivity (Sn), Specificity (Sp), Positive Predictive Value (PPV) and Negative Predictive Value (NPV) in the assessment of high grade dysplasia vs. no dysplasia with white-light endoscopy and optical magnification (WLE-z), narrow-band imaging and optical magnification (NBI-z) and a combination of both ( $95 \%$ confidence interval).

\begin{tabular}{|lllll|}
\hline & Sn, \% & Sp, \% & PPV, \% & NPV, \% \\
\hline WLE-z & 48.4 & 92.2 & 45.6 & 93.5 \\
& $(45.3-50.6)$ & $(90.5-93.4)$ & $(42.3-47.6)$ & $(91.6-94.3)$ \\
\hline NBI-z & 89.1 & 89.3 & 56.5 & 98.3 \\
& $(87.3-90.6)$ & $(87.3-90.6)$ & $(53.4-58.6)$ & $(97.2-98.7)$ \\
\hline $\begin{array}{l}\text { Combi- } \\
\text { nation }\end{array}$ & 93.4 & 90.3 & 61.7 & 99.2 \\
\hline & $(91.6-94.3)$ & $(88.4-91.5)$ & $(58.4-63.5)$ & $(98.4-99.5)$ \\
\hline
\end{tabular}

Table 3 Narrow-band imaging (NBI) experience of participants and accuracy in detecting dysplasia (95\% confidence interval).

\begin{tabular}{|lll|}
\hline & $\begin{array}{l}\text { Not experienced with NBI, } \\
\%\end{array}$ & $\begin{array}{l}\text { Experienced with NBI, } \\
\%\end{array}$ \\
\hline WLE-z & $86.7(84.2-88.9)$ & $88.3(84.0-91.8)$ \\
\hline NBI-z & $87.9(85.5-90.0)$ & $90.9(87.2-93.8)$ \\
\hline Combination & $89.0(86.7-91.9)$ & $94.6(91.0-97.0)$ \\
\hline
\end{tabular}

WLE-z, white-light endoscopy with magnification; NBI-z, narrow-band imaging with magnification.

Table 4 Participant experience with optical magnification and accuracy in detecting dysplasia ( $95 \%$ confidence interval).

\begin{tabular}{|c|c|c|}
\hline & $\begin{array}{l}\text { Not experienced with } \\
\text { optical magnification, \% }\end{array}$ & $\begin{array}{l}\text { Experienced with } \\
\text { optical magnification, } \\
\%\end{array}$ \\
\hline WLE-Z & $86.4(83.2-89.2)$ & $87.7(84.8-90.3)$ \\
\hline NBI-Z & $88.5(85.6-91.0)$ & $88.8(86.1-91.2)$ \\
\hline Combination & $87.3(83.9-90.2)$ & $92.7(90.4-94.7)$ \\
\hline
\end{tabular}

WLE-z, white-light endoscopy with magnification; NBI-z, narrow-band imaging with magnification.

Image evaluation: by experience with NBI and optical magnification

Twenty participants $(71 \%)$ routinely use NBI in daily practice. The overall Acc in detecting dysplasia in BE for experienced endoscopists was $95 \%$ in contrast to $89 \%$ for endoscopists not experienced with NBI when both modalities were used $(P<0.05)$ ( $\bullet$ Table 3). Fourteen participants ( $50 \%$ ) have experience with optical magnification technology. An Acc of $93 \%$ was achieved by endoscopists with experience in optical magnification when the combined modalities of NBI-z and WLE-z were used compared to $87 \%$ with endoscopists who were not experienced with this technique $(P<0.05)(\odot$ Table 4$)$. 
Intraobserver agreement

The intraobserver agreement (kappa) for WLE-z, NBI-z, and the combination was $0.31,0.49$, and 0.56 , respectively.

\section{Discussion}

This study looked at two endoscopic imaging modalities diagnosing dysplasia in patients who had BE. Current surveillance and practice guidelines for BE are not widely followed as there is a marked variability in the technique and interval of surveillance $[10,11]$. The identification of dysplasia can be difficult and distribution is multifocal leading to sampling error in even the most thorough biopsy surveillance protocols [12]. The American Society of Gastrointestinal Endoscopy's Preservation and Incorporation of Valuable endoscopic Innovations guidelines [13] on imaging technology in $\mathrm{BE}$ recommend that an imaging technology with targeted biopsies should have a per-patient sensitivity of $90 \%$ or greater and a negative predictive value (NPV) of $98 \%$ or greater for detecting high grade dysplasia/early esophageal adenocarcinoma. In the present study, a high NPV of $99 \%$ could be achieved when WLE-z and NBI-z were used, although it must be taken into context that this was an analysis performed on a perimage rather than a per-patient basis.

NBI with optical magnification has been extensively studied in the past with a myriad of different classification systems [1416]. Baldaque-Silva et al. [17] recently concluded that structured learning in endoscopic assessment of $\mathrm{BE}$ using magnification and NBI did not have an impact on accuracy based on the Amsterdam classification system. The authors obtained sensitivity for prediction of neoplasia ranging between $62 \%$ and $90 \%$. The same group also compared three different classification systems (Nottingham, Kansas, and Amsterdam) for BE using NBI and magnification with nine endoscopists from Europe and Japan [18]. The global accuracy for detection of non-dysplastic and dysplastic lesions using these three classifications was rather disappointing at $46 \%, 47 \%$, and $51 \%$, respectively. Our study differs from that of the above as a simplified classification system was used. The evaluators in this study also had a learning phase and appeared to be well versed with the classification system before embarking on the online module, although the image acquisition process and selection bias as described previously could have also led to the higher NPV in this study.

This study has some limitations. A single endoscopist with extensive experience in mucosal imaging obtained all of the images. This may not be generalizable to the community Gastroenterologist. However, with the advent of newer endoscopic imaging devices, obtaining clear, crisp images is becoming increasingly easier. Another drawback is that the images were evaluated 'post hoc' and not in real time. There were also no low grade dysplastic (LGD) lesions evaluated in the study. This was deliberately done as NBI-z does not reliably diagnose LGD. It is well known though that there is significant interobserver variability in the diagnosis of LGD even amongst expert gastrointestinal pathologists [19]. In conclusion, this study demonstrated that a high NPV in the prediction of non-dysplastic areas in BE can be achieved with the application of a combination of WLE-z and NBI-z. It may thus be plausible to refrain from biopsying regular appearing areas, resect irregular appearing areas (both of which when diagnosed with high confidence and performed at skilled resourced center), and biopsy only equivocal areas. This cost and time saving approach could potentially lead to a paradigm shift in how patients with $B E$ are surveyed. Real-time prospective evaluation of dysplasia in BE will further consolidate and support the findings of this study.

\section{Competing interests: None}

\section{Institutions}

${ }^{1}$ Lyell McEwin Hospital, Adelaide, Australia

2 University of Adelaide, Adelaide, Australia

${ }^{3}$ Department of Medicine, Yong Loo Lin School of Medicine, National University of Singapore, Singapore

${ }^{4}$ Division of Gastroenterology, The Second Affiliated Hospital, Xi'an Jiaotong University, Xi'an, China

${ }^{5}$ Department of Internal Medicine, National Taiwan University Hospital, Taipei, Taiwan

${ }^{6}$ Department of Gastroenterology and Hepatology, Singapore General Hospital, Singapore

${ }^{7}$ Asian Institute of Gastroenterology, Hyderabad, India

${ }^{8}$ Osaka Medical Center for Cancer and Cardiovascular Diseases, Osaka, Japan

${ }^{9}$ Division of Gastroenterology and Hepatology, Department of Medicine,

Faculty of Medicine, University of Malaya, Kuala Lumpur, Malaysia

${ }^{10}$ Institute of Endoscopy, Zhongshan Hospital, Shanghai, China

${ }^{11}$ Department of Gastroenterology, Seth GS Medical College and KEM Hospital, Mumbai, India

12 Department of Endoscopy, Nagoya University Hospital, Nagoya City, Aichi, Japan

${ }^{13}$ Department of Gastroenterology, Nagoya University Graduate School of Medicine, Nagoya City, Aichi, Japan

14 Institute of Digestive Disease, Department of Medicine \& Therapeutics, Prince of Wales Hospital, The Chinese University of Hong Kong, Shatin, Hong Kong

${ }^{15}$ Department of Internal Medicine and Gastroenterology, Cipto Mangunkusumo Hospital, Jakarta, Indonesia

${ }^{16}$ Department of Internal Medicine, E-Da Hospital/I-Shou University, Kaohshiung, Taiwan

17 Department of Gastroenterology, Asan Medical Center, Seoul, Korea

${ }^{18}$ Department of Internal Medicine, Padjadjaran University, Bandung, Indonesia

${ }^{19}$ Department of Gastroenterology, Fukuoka University, Chikushi Hospital, Fukuoka, Japan

20 Department of Gastroenterology, RIPAS Hospital, Brunei Darussalam

${ }^{21}$ Division of Gastroenterology and Hepatology, Veterans Affairs Medical Centre and University of Kansas School of Medicine, Kansas City, MO, United States

\section{References}

1 Conteduca $V$, Sansonno $D$, Ingravallo $G$ et al. Barrett's esophagus and esophageal cancer: an overview. Int J Oncol 2012; 41: 414-424

2 Falk GW, Ours TM, Richter JE. Practice patterns for surveillance of Barrett's esophagus in the United States. Gastrointest Endosc 2000; 52: 197-203

3 Jayasekera C, Taylor AC, Desmond PV et al. Added value of narrow band imaging and confocal laser endomicroscopy in detecting Barrett's esophagus neoplasia. Endoscopy 2012; 44: 1089-1095

4 Allmond LM, Barr H. Advanced endoscopic imaging in Barrett's oesophagus. Int J Surg 2012; 10: 236-241

5 Lee MH, Buterbaugh K, Richards-Kortum R et al. Advanced endoscopic imaging for Barrett's oesophagus: current options and future directions. Curr Gastroenterol Rep 2012; 14: 216-225

6 Curvers WL, van Vilsteren FG, Baak LC et al. Endoscopic trimodal imaging versus standard video endoscopy for detection of early Barrett's neoplasia: a multicentre, randomised crossover study in general practice. Gastrointest Endosc 2011; 73: 195-203

7 Wang KK, Okoro N, Prasad G et al. Endoscopic evaluation and advanced imaging of Barrett's esophagus. Gastrointest Endosc Clin N Am 2011; 21: $39-51$

8 Sharma P, Bansal A, Mathur $S$ et al. The utility of a novel narrow band imaging endoscopy system in patients with Barrett's esophagus. Gastrointest Endosc 2006; 64: 167-175

9 Sharma P, Hawes RH, Bansal A et al. Standard endoscopy with random biopsies versus narrow band imaging targeted biopsies in Barrett's oesophagus: a prospective, international, randomised controlled trial. Gut 2013; 62: 15-21

10 Gross CP, Canto MI, Hixson J et al. Management of Barrett's esophagus: a national study of practice patterns and their cost implications. Am J Gastroenterol 1999; 94: 3440 - 3447 
11 Das D, Ishaq S, Harrison R et al. Management of Barrett's esophagus in the UK: overtreated and underbiopsied but improved by the introduction of a national randomized trial. Am J Gastroenterol 2008; 103: $1079-1089$

12 Abrams JA, Kapel RC, Lindberg GM et al. Adherence to biopsy guidelines for Barrett's esophagus surveillance in the community setting in the United States. Clin Gastroenterol Hepatol 2009; 7: 736 - 742

13 Sharma P, Savides TJ, Canto MI et al. The American Society for Gastrointestinal Endoscopy PIVI (Preservation and Incorporation of Valuable Endoscopic Innovations) on imaging in Barrett's Esophagus. Gastrointest Endosc 2012; 76: 252 - 254

14 Kara MA, Ennahachi M, Fockens $P$ et al. Detection and classification of the mucosal and vascular patterns (mucosal morphology) in Barrett's esophagus by using narrow band imaging. Gastrointest Endosc 2006; 64: $155-166$

15 Sharma P, Bansal A, Mathur $S$ et al. The utility of a novel narrow band imaging endoscopy system in patients with Barrett's esophagus. Gastrointest Endosc 2006; 64: 167-175
16 Singh R, Anagnostopoulos GK, Yao K et al. Narrow Band Imaging with magnification in Barrett's esophagus: validation of a simplified grading system of mucosal morphology patterns against histology. Endoscopy 2008; 40: $457-463$

17 Baldaque-Silva F, Marques $M$, Lunet $N$ et al. Endoscopic assessment and grading of Barrett's esophagus using magnification endoscopy and narrow band imaging: impact of structured learning and experience on the accuracy of the Amsterdam classification system. Scand J Gastroenterol 2013; 48: 160 - 167

18 Silva FB, Dinis-Ribeiro $M$, Vieth $M$ et al. Endoscopic assessment and grading of Barrett's oesophagus using endoscopy and narrow-band imaging: accuracy and interobserver agreement of different classification systems (with videos). Gastrointest Endosc 2011; 73: 7-14

19 Wani S. Management of low-grade dysplasia in Barrett's oesophagus. Curr Opin Gastroenterol 2012; 28: 370-376 